\title{
Oxigenación con membrana extracorpórea
}

\section{Extracorporeal membrane oxigenation}

Rafael Lima Linares', Manuel Alberto Guerrero Gutiérrez², Vázquez Peralta³, Arturo Pérez Nieto ${ }^{4}$, Rubén Orlando ${ }^{5}$, Eder Iván Zamarrón-López ${ }^{6}$, Raúl Soriano Orozoco ${ }^{7}$, Marco Antonio Montes de Oca Sandoval ${ }^{8}$, Carlos Riera Kinkel ${ }^{8}$, Edgar Hernández Réndon ${ }^{9}$, David Roldan Morales ${ }^{10}$, Antonio Fernández Reyes ${ }^{11}$, Rene D. Gómez Gutiérrez ${ }^{11}$

\begin{abstract}
Extra-corporeal membrane oxygenation (ECMO) is a strategy to maintain the proper gas exchange that is commonly used during cardiopulmonary surgeries, whose current tendency is to increase the spectrum of acute diseases outside the operating room that significantly compromise the oxygenation, ventilation or circulation. This therapy supplies the heart, lungs, or both, totally or partially, and depending on its configuration, it can be applied to improve respiratory (venous-venous), circulatory (venous-arterial ECMO) or both, providing time for Damaged tissue recovers. ECMO is a therapy commonly used in North America and Europe, however, in Mexico it is just beginning to be part of a promising future.
\end{abstract}

\section{Key words:}

ECMO, cardiopulmonary resuscitation, venous-venous, venous-arterial

\footnotetext{
Anestesiólogo Cardiovascular adscrito al Servicio de Cirugía Cardiovascular en el Hospital de Cardiología, CMN Siglo XXI, IMSS.

2 Médico residente de primer año en Medicina critica. Instituto Nacional de Cancerología. Ciudad de México, México.

3 Médico residente en Anestesiología Cardiovascular. Instituto Nacional de Cardiología "Ignacio Chávez". Ciudad de México, México.

4 Especialista en Urgencias Médico-Quirúrgicas y Terapia intensiva. Hospital General de San Juan del Río. Querétaro, México.

5 Especialista en Urgencias Médico-Quirúrgicas y Terapia intensiva. Hospital CEMAIN, Tampico. Tamaulipas, México.

6 Especialista en Urgencias Médico-Quirúrgicas y Terapia intensiva. UMAE T1, CMN Bajío. León, Gto.

7 Especialista en Urgencias Médico-Quirúrgicas y Terapia intensiva. Centro Médico ABC. Ciudad de México, México.

8 Cirujano Cardiovascular. UMAE Cardiología, Centro Médico Nacional Siglo XXI. Ciudad de México, México.

9 Cirujano Cardiovascular. UMAE Cardiología. Mérida, Yucatán.

10 Médico General. Practica privada. Ciudad de México, México.

11 Médico especialista en Pediatría. Hospital Christus Muguerza, vicepresidente ELSO LATAM. Monterrey, Nuevo León.
}

Fecha de ingreso: 28 de septiembre de 2020

Fecha de aceptación: 23 de octubre de 2020

\section{ORCID}

0000.0002-0645-1836

Conflicto de Interés

Todos los autores declaran no tener conflicto de interés.

Financiamiento

Los autores no recibieron financiamiento de ningún tipo para la realización del manuscrito.

\section{Correspondencia:}

Guerrero Gutiérrez Manuel Alberto

Manuelguerreromd@gmail.com 


\section{RESUMEN}

La oxigenación por membrana extra corpórea (ECMO por sus siglas en inglés), es una estrategia para mantener el adecuado intercambio gaseoso que se utiliza habitualmente durante cirugías cardiopulmonares, cuya tendencia actual es al incremento del espectro de enfermedades agudas fuera de quirófano que comprometen significativamente la oxigenación, la ventilación o la circulación. Esta terapia suple total o parcialmente al corazón, a los pulmones, o ambos, y dependiendo de su configuración, puede ser aplicado para mejorar la función respiratoria (ECMO veno-venoso), circulatoria (ECMO veno-arterial) o ambas, proporcionando tiempo para que el tejido dañado se recupere. El ECMO es una terapia de uso habitual en Norteamérica y Europa, sin embargo, en México apenas comienza a ser parte de un futuro prometedor.

\author{
Palabras clave: \\ ECMO, \\ resucitación \\ cardiopulmonar, \\ veno-venoso, \\ veno-arterial
}

\section{Introducción}

a oxigenación por membrana extra corpórea (ECMO por sus siglas en inglés), ha presentado un avance impresionante en los últimos años en México, convirtiéndose en una pieza invaluable en el manejo del paciente crítico, tanto en adultos como en niños, con severo daño pulmonar y/o cardiaco, refractario a tratamiento convencional[1],[2].

Hoy en día la terapia ECMO se ha convertido en un equipo de mayor confianza con sus distintas configuraciones como es el ECMO veno-venoso y veno-arterial, teniendo indicaciones con un buen nivel de evidencia, como son: puente a recuperación cardiaco y/o pulmonar, puente a trasplante cardiaco y/o pulmonar, paradaa cardiaca, resecciones pulmonares en pacientes inestables[3]-[14]. La decisión de iniciar la terapia de ECMO en un paciente grave, debe ser realizada por personal capacitado y entrenado en esta área, así como el mantenimiento y termino del mismo[15].

\section{Antecedentes}

En 1944, Kolff y Berk[16] notaron que la sangre se oxigenaba a través de las cámaras de celofán del riñón artificial en pacientes con necesidad de sustitución de la función renal, este concepto fue aplicado en 1953 por Gibbon quien uso perfusión y oxigenación artificial, para apoyar con éxito una cirugía a corazón abierto[17]. En 1965, Rashkind y sus colaboradores fueron los primeros en utilizar un oxigenador de burbuja como apoyo para un neonato con insuficiencia respiratoria grave[18]. En el año 1969, Dorson y colaboradores, reportaron con éxito el uso de una membrana de oxigenación para un bypass cardiopul- monar en pacientes pediátricos[19]. En 1970, Baffles y colaboradores, reportan el uso exitoso de una oxigenación por membrana extracorpórea, en pacientes pediátricos con defectos cardiacos congénitos, que se sometieron a cirugía cardiaca[20].

La terapia de ECMO de "largo plazo", se utilizo para brindar apoyo a los pacientes con Síndrome de Distrés Respiratorio del Adulto (SDRA), siendo usado exitosamente por primera vez en 1972, en un paciente adulto con poli trauma e insuficiencia respiratoria[21]. En ese mismo año, Zapol, considerado uno de los padres del ECMO veno-venoso, publicpo en el New England Journal of Medicine, que la meta del Extra corporeal life support (ECLS), es comprar tiempo, mientras se resuelve la causa y se obtiene una adecuada perfusión[22].

Kolobow y Gattinoni en 1977 desarrollaron una nueva membrana pulmonar para mejorar el intercambio de dióxido de carbono $\left(\mathrm{CO}_{2}\right)$ y removerlo más rápidamente $\left(\mathrm{ECCO}_{2} \mathrm{R}\right)$, como una posible opción en el paciente con enfermedad pulmonar obstructiva cróniCa (EPOC)[23]. En 1975, Barlett et al., reportó por primera vez exitosamente el uso de ECMO en neonatos con insuficiencia respiratoria severa[24].

La trascendencia de la terapia de ECMO se inició en 1989 en Nueva Orleans, debido al interés y la necesidad de coordinar los centros especializados de ECMO, por lo que se crea ELSO (extracorporeal life support organization) con el fin de estandarizar los procesos de la terapia y recolectar los datos obtenidos para futuras investigaciones.

A inicios de los años 90, Morris et al., fallaron al mostrar alguna ventaja adicional al implementar el ECMO versus la terapia convencional de ventilación mecánica en SDRA, en un estudio aleatorizado[25]. A pesar de la falta de evidencia, algunos centros en 
Estados Unidos y Europa continuaron colocando el ECMO veno-venoso, apoyado en la terapia de ventilación mecánica en pacientes seleccionados, sin embargo, con el sesgo de iniciarlo tardíamente al considerarse una terapia de "último recurso"[26]-[28]. El uso de ECMO repunto durante la pandemia de influenza AH1N1 en el 2009 y posterior a los estudios CESAR y posteriormente EOLIA, donde no se demostró una mejora en la sobrevida, pero tampoco un daño asociado al uso de esta terapia, y la discapacidad a los 6 meses, los estudios fueron randomizados en pacientes con SDRA que fallaron al tratamiento convencional, comparados con centros donde no se ofreció la terapia ECMO[3]-[29].

\section{Ecmo veno-venoso}

El ECMO veno-venoso (VV), es caracterizado por la oxigenación de la sangre, así como por la eliminación del $\mathrm{CO}_{2}$ en la sangre venosa, es decir es utilizado para tratar la falla pulmonar pura. La patología por excelencia, donde se encuentra hipoxemia severa y retención de $\mathrm{CO}_{2}$, es el SDRA. Ashbaugh y colaboradores describieron en 1967 un reporte de 12 casos que describe la presentación clínica de pacientes críticos con hipoxemia refractaria, edema pulmonar no cardiogénico, aumento de trabajo respiratorio, opacidades bilaterales en la radiografía de tórax, secundario a una infección por trauma requiriendo de ventilación mecánica[30], fallecieron 7 pacientes de los 12, pero en 6 se encontró abundante membrana hialina en el espacio alveolar compatible con el Síndrome de Distrés Respiratorio del recién nacido, posteriormente el termino cambio a ser del adulto, luego a agudo y finalmente como Síndrome de Distrés Respiratorio Agudo[31]. En 1994, se le otorgo el nombre de SDRA durante la Conferencia de Consenso Americano y Europeo (AECC), tomando en cuenta el tiempo de inicio (Agudo), rayos $X$ de tórax, edema no cardiogénico y $\mathrm{PaO}_{2} / \mathrm{FiO}_{2}$ de $300-200 \mathrm{mmHg}$, posteriormente en el 2012 en el panel de expertos de la sociedad europea de medicina cuidado intensivo se estableció la definición de Berlín la cual propone, un inicio agudo en los primeros 7 días, radiográficamente presencia de opacidades bilaterales no explicados por derrame pleural, atelectasias o nódulos, insuficiencia respiratoria no atribuible a falla cardiaca o sobrecarga hídrica e índice $\mathrm{P} / \mathrm{F}\left(\mathrm{paO}_{2} / \mathrm{FiO}_{2}\right)$ de 300-200 mmHg para SDRA leve, 200-100 mm Hg moderado y menor de 100 mmHg Severo con PEEP mayor o igual de $5 \mathrm{~cm} \mathrm{H}$ O (Berlín 2012)[32]. Actualmente, lo podemos definir de forma simplificada de la siguiente manera (Tabla 1).

La eficacia del sistema de oxigenación de membrana extracorpórea veno-venoso, en pacientes con SDRA ha permanecido en controversia, pero las ultimas revisiones sistematizadas y metaanálisis, han dado sustento al uso de la terapia de ECMO[33],[34].

Los pacientes que han recibido la terapia de ECMO durante las pandemias de influenza A (H1N1) en el 2009, han tenido resultados prometedores. El ECMO fue diseñado para limitar el daño y rescatar el pulmón, determinando el inicio de la terapia temprana de ECMO en los casos de SDRA severo[35]-[37].

El estudio EOLIA de 2018, es el estudio más grande con uso de terapia ECMO veno-venoso en el SDRA severo comparando 2 grupos, ambos con SDRA severo, uno se le colocaba el ECMO veno-venoso (específicamente $\mathrm{Pa} / \mathrm{FiO}_{2}$ menor a $50 \mathrm{mmHg}$ por $3 \mathrm{~h} \mathrm{o} \mathrm{Pa} /$ $\mathrm{FiO}_{2}$ menor a $80 \mathrm{mmHg}$ por $6 \mathrm{~h} \mathrm{o} \mathrm{pH}$ arterial menor a 7,25 con una presión parcial de $\mathrm{PaCO}_{2}$ mayor a 60 $\mathrm{mmHg}$ por $6 \mathrm{~h}$, siendo la meta primaria la mortalidad a 60 días[38] (Tabla 2).

Se recomienda el uso del prono previo a la terapia de ECMO en el SDRA severo[39] (Figura 1) siendo un grupo considerable, entonces la reformulando la pregunta inicial, quedaría planteada de esta manera ¿Qué tanto me va beneficiar el ECMO en esta terapia y a que costo? Consideramos que el uso de ECMO no debería limitarse a aquellos pacientes con SDRA severo, debido a que la mortalidad de la patología per se, se encuentra incrementada, una intervención temprana de esta terapia disminuiría la mortalidad en este tipo de pacientes, aunque actualmente solo se cuente como terapia de "rescate", en un futuro no muy lejano, el ECMO será un pilar fundamental en el manejo temprano del SDRA.

\section{Tabla 1. Criterios de Berlín para el SDRA[32]}

Infiltrados pulmonares bilaterales en estudio de imagen (radiografía de tórax, tomografía computarizada o ultrasonido pulmonar)

Edema pulmonar no justificado principalmente por falla cardiaca (evidenciado de forma objetiva, ej. Ecocardiografía)

$\mathrm{PaO}_{2} / \mathrm{FiO}_{2}<300$ (Con PEEP $\geq 5 \mathrm{cmH}_{2} \mathrm{O}$ )

Afección pulmonar de inicio menor a 7 días 


\section{Tabla 2. Indicaciones de ECMO Veno-venoso}

SDRA severo con $\mathrm{Pa} / \mathrm{FiO}_{2}$ menor a $50 \mathrm{mmHg}$ por $3 \mathrm{~h}$

$\mathrm{Pa} / \mathrm{FiO}_{2}$ menor a $80 \mathrm{mmHg}$ por $6 \mathrm{~h}$

$\mathrm{pH}$ arterial menor a 7,25 con una presión parcial de $\mathrm{PaCO}_{2}$ mayor a $60 \mathrm{mmHg}$ por $6 \mathrm{~h}$

\section{Contraindicaciones del ECMO veno-venoso}

\section{Absolutas[40]}

- Paciente moribundo con una falla orgánica múltiple establecida.

- Pacientes con mal pronóstico a corto plazo (ej. Enfermedad metastásica maligna).

- Comorbilidades en etapa avanzada, como; insu- ficiencia respiratoria crónica sin indicación para trasplante o irreversible.

- Patología neurológica devastadora (ej. Hemorragia masiva intracraneal).

\section{Relativas[40]}

- Ventilación mecánica con presiones altas, por más de 7 días.

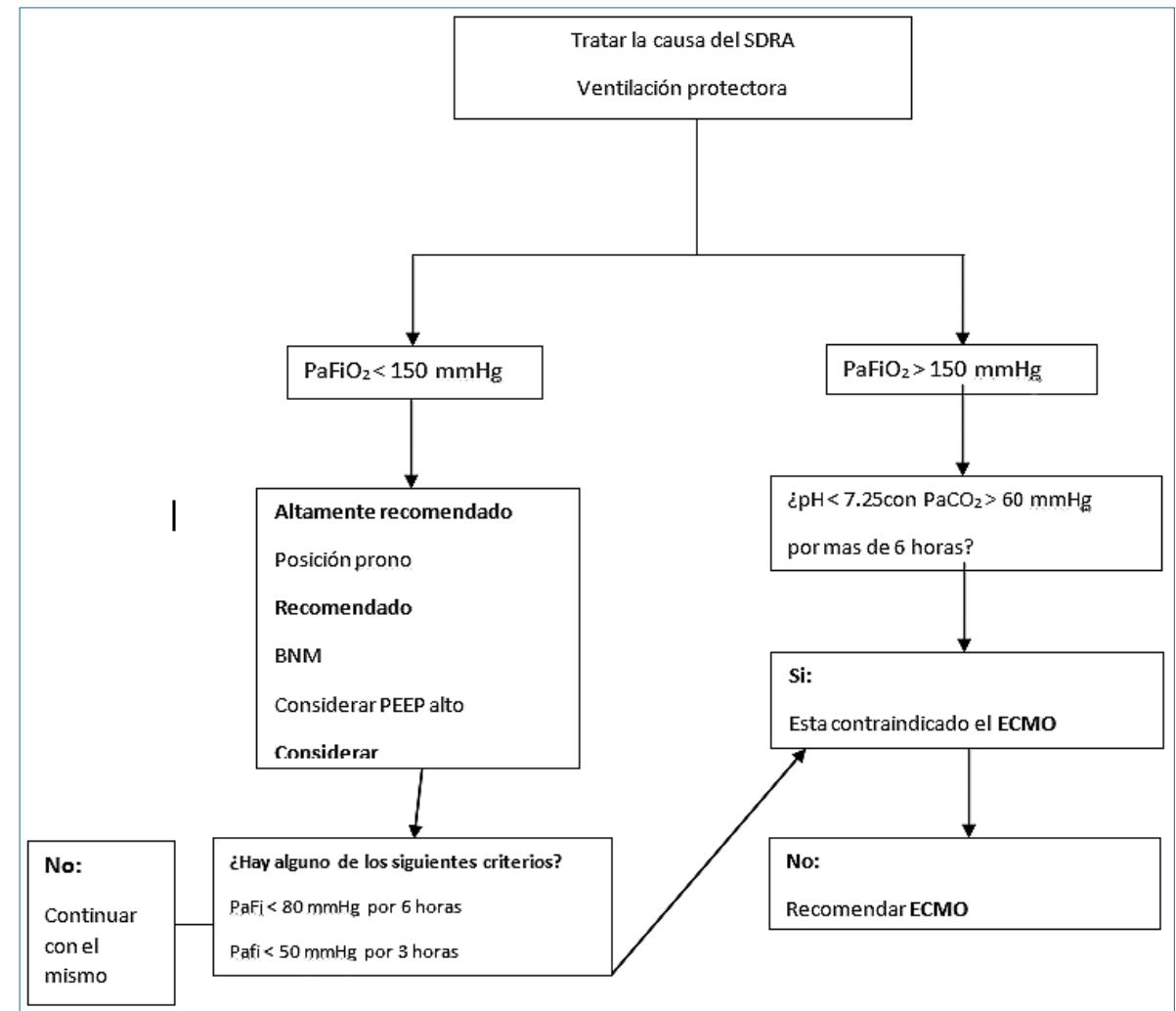

Grafico 1. Algoritmo de manejo para SDRA. PEEP = Presión positive al final de la espiración; $\mathrm{PaO}_{2}: \mathrm{FiO}_{2}=$ Índice de presión arterial de oxígeno en sangre arterial y fracción inspirada de oxígeno: $\mathrm{ECMO}=$ Membrana extracorpórea de oxigenación; $\mathrm{PaCO}_{2}$ = Presión parcial de dióxido de carbono en sangre arterial. ${ }^{*}$ Con respiración mayor a 35 ventilaciones por minute y ventilación mecánica a una presion plateau ajustada $\leq 32 \mathrm{~cm}$ de agua. + Considerar bloqueo neuromuscular. No hay contraindicaciones absolutas, excepto estadio final con falla respiratoria, cuando no ha sido considerado el trasplante pulmonar. Ej, Bloqueo neuromuscular, PEEP alto, vasodilatadores pulmonares inhalados, maniobras de reclutamiento alveolar, ventilación oscilatoria de alta frecuencia. Se recomienda terapia temprana con ECMO con base en el estudio EOLIA. **Considerar en cada hospital la logística necesaria, para tener un ECMO, sugerimos que al considerar posición prono, se active la alerta de ECMO para iniciar la logística y tener un ECMO Team disponible al ser necesario. 
- Edad avanzada.

- Accesos vasculares limitados.

- Sangrado activo.

- Contraindicaciones para limitar la anticoagulación (en ciertas ocasiones, se puede colocar sin anticoagulación).

\section{Centros ECMO}

Desde que se comenzó a utilizar el ECMO para los pacientes con SDRA, los centros han reportado resultados muy heterogéneos[41], se consenso que el volumen anual para un centro de ECMO, debe ser, de al menos 20 casos al año, con un mínimo de 12 casos al año para falla respiratoria aguda[42]. En caso de no contar con esta experiencia, los pacientes deben ser referidos a un centro de ECMO capacitado, con el objetivo de disminuir complicaciones.

\section{Complicaciones}

Las complicaciones durante el ECMO, son comunes y potencialmente ponen en riesgo la vida, se describen en la Tabla 3, las cuales son menos frecuentes cuando la terapia es otorgada por un equipo entrenado y es realizada en un centro especializado. Es importante reconocerlas y tratarlas a la brevedad posible.

\section{ECMO venoarterial}

\section{Introducción}

La oxigenación con membrana extracorpórea Ve- noarterial (ECMO-VA) provee de un soporte cardiopulmonar para pacientes con choque cardiogénico multifactorial con falla uni o biventricular y/o disfunción pulmonar, choque post cardiotomía, como terapia puente de recuperación miocárdica, soporte mecánico circulatorio para trasplante cardiaco; en el contexto de los avances en tecnología de membranas, bombas, estrategias de canulación percutánea y la adecuada selección de pacientes[51].

Con el paso de los años, se han desarrollado nuevos elementos, como membranas de fibra en tubos huecos que disminuyen la resistencia en el oxigenador y mejoran las características de compatibilidad de la sangre, bombas centrifugas rediseñadas que disminuyen la generación de calor y trombogenicidad, permitiendo un soporte más duradero y mas biocompatible, configuraciones hibridas de ECMO VAV (Veno-arterio-venoso) que proveen flujo sanguíneo pulmonar supraoxigenado, mientras da aporte hemodinámico al mismo tiempo, desplegar estrategias percutáneas y circuitos a menor escala facilitando el trasporte del paciente con Ecmo, todo ello permitiendo acercarse la utopía que en los años 30's se empezaba a proyec$\operatorname{tar[52].~A~pesar~de~todos~los~avances~tecnológicos;~la~}$ supervivencia a 6 meses de los pacientes con ECMO VA sigue siendo del 30\% y una mortalidad hospitalaria del $50 \%$ a $60 \%$ quedando supeditada la necesidad de desarrollo e investigación en este campo. En un análisis a 15 años del registro Extracorporeal Life Support Organization (ELSO) se reportó una sobrevida de tan solo del $15 \%$.

En la última década los centros de ECMO-VA han incrementado significativamente el número de pacientes, Gerke[53] y cols., describieron que en EU se

\section{Tabla 3.}

\begin{tabular}{ll}
\hline Complicaciones & Consideraciones \\
Canulación[43]-[45] & ELSO reporta 6\% de complicaciones relacionadas a la canulación \\
& Debe ser realizada por un operador experto, usando ultrasonido o fluoroscopia \\
& Las complicaciones asociadas a la canulación, son potencialmente mortales por lo que se debe de \\
& disponer de hemoderivados \\
& Se debe fijar adecuadamente la cánula, para evitar migración \\
& Embolismo aéreo es reportado, por lo que debe ser de estrecha vigilancia \\
& Infección asociada al sitio de canulación \\
& ELSO reporta 10\% de complicaciones asociadas a fallos en la tecnica y el oxigenador \\
Técnica[46],[47] & Las membranas de polimetilpentano y las bombas de la centrifuga en circuitos modernos, prác- \\
& ticamente han eliminado la fuga de plasma, calentamiento de la cabeza de la bomba y ruptura \\
& de la tubería \\
Trombosis y hemorra- & ELSO reporta 3,8\% de sangrado intracerebral en pacientes adultos con ECMO V-V \\
gia[48]-[50] & Hemolisis mínima es observada durante el ECMO V-V \\
& Activación y destrucción plaquetaria, es uno de los riesgos por sangrado en ECMO
\end{tabular}

ELSO: Extracorporeal life support; ECMO-VV: Extracorporeal membrane oxygenation venovenous. 
han registrado 12.566 pacientes adultos en el programa Extracorporeal Life Support Organization.

\section{ECMO venoarterial}

El ECMO VA consiste en un circuito donde la sangre es drenada desde la aurícula derecha, a través de una cánula inserta en la vena yugular interna derecha, vena femoral o directamente en la aurícula derecha y es retornada a la aorta torácica, a través de una cánula carotídea derecha, femoral o aórtica (Figura 2). La principal meta de la modalidad VA es la restauración de la perfusión tisular para permitir la estabilización y recuperación funcional[51]-[59] (Tabla 4).

A pesar de que no existe suficiente bibliografía, que sustente recomendaciones específicas del manejo de ECMO VA se propone como meta inicial que el flujo debería ser de 50-70 ml/kg/min con una presión arterial media $>60 \mathrm{mmHg}$ y una presión diferencial de mas de $10 \mathrm{mmHg}[51],[60]$. Idealmente se debería colocar línea arterial radial en el brazo derecho, con el fin de que las muestras tomadas para análisis de gases sanguíneos se correlacionen mejor con el contenido cerebral de oxígeno; además permite monitorizar la presión de pulso (pulsatilidad arterial) como medida indirecta de contractilidad cardiaca durante el soporte y destete, una pulsatilidad bajo o nulo $(<10 \mathrm{mmHg})$ indica que el ventrículo izquierdo no está eyectando el suficiente volumen generando estasis sanguínea y mayor riesgo de formación trombótica; presión de pulso elevada indica posible recuperación miocárdica. Potenciales fuentes de formación de trombos por estasis sanguínea son el VI, la raíz aortica e incluso el sistema del ECMO, por lo tanto, el manejo anticoagulante es indispensable bajo medidas farmacológicas y no farmacológicas[59]. Las no farmacológicas implican la evaluación visual directa del circuito y el oxigenador de membrana, monitoreo de las presiones ejercidas en el circuito e indirectamente evaluando las medidas de hemolisis (niveles de LDH y hemoglobina libre de plasma). Bivalirudina y Argatroban se han reportado como alternativas seguras y efectivas para la anticoagulación en pacientes con trombocitopenia inducida por heparina o resistencia a la heparina, la heparina no fraccionada es el anticoagulante de elección[60].

Los niveles de anticoagulación para ECMO VA son más altos que para ECMO VV por la naturaleza fatídica de la trombosis sistémica o el colapso circulatorio que puede ocurrir por un fallo en el circuito. En 2015 Silvetti[61] estudió circuitos recubiertos con heparina para minimizar formación de microtrombos y para reducir la dosis de heparina sistémica con resultandos

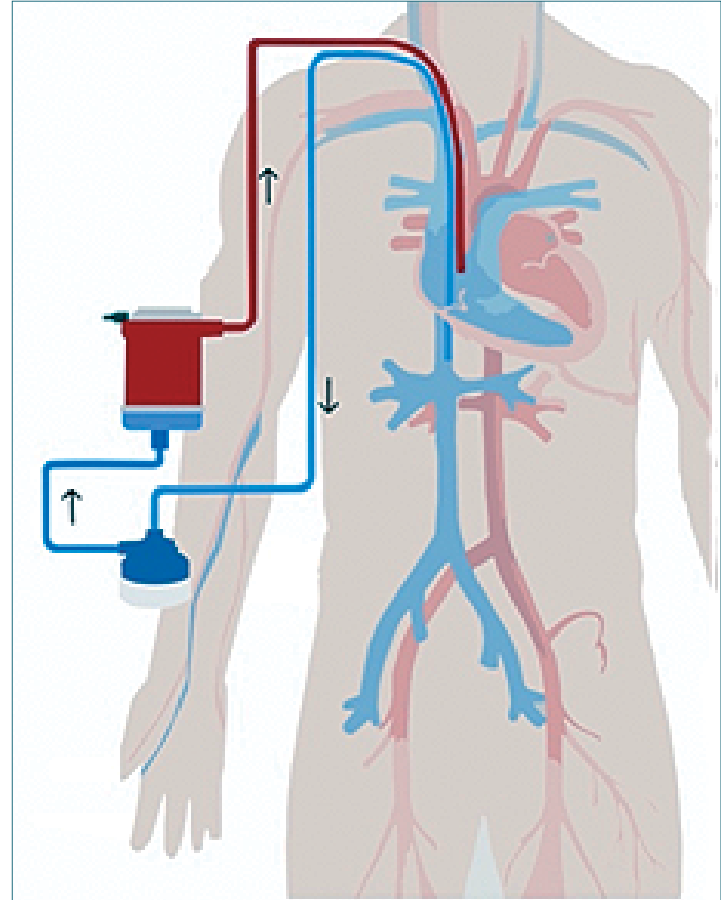

Figura 1. ECMO Veno-venoso.

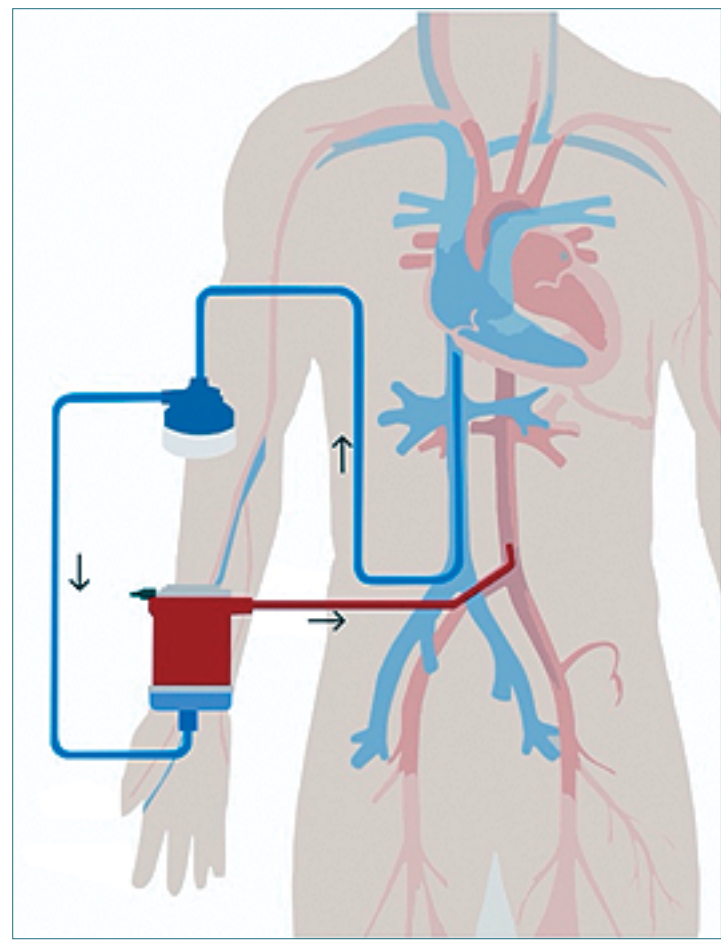

Figura 2. ECMO veno-arterial. 


\section{Tabla 4. Objetivos principales del ECMO VA}

\begin{tabular}{ll}
\hline Contexto & Descripción \\
Enlace hacia la recuperación & $\begin{array}{l}\text { Temporizar el soporte circulatorio mientras se implementan estrategias para restaurar la } \\
\text { recuperación miocárdica y lograr un destete exitoso }\end{array}$ \\
Enlace hacia la decisión & $\begin{array}{l}\text { Para determinar la reversibilidad del daño del órgano terminal comúnmente visto después } \\
\text { de un evento critico miocárdico para decidir el siguiente nivel de acción }\end{array}$ \\
"Bridge to Bridge" & $\begin{array}{l}\text { Para lograr una breve estabilidad para la perfusión del órgano terminal hasta que se inicie } \\
\text { el soporte circulatorio mecánico duradero o la terapia de reemplazo cardiaco sea realizada }\end{array}$ \\
Enlace hacia el trasplante & $\begin{array}{l}\text { Para lograr una breve estabilidad para la perfusión del órgano terminal hasta que se realice } \\
\text { el trasplante de corazón }\end{array}$ \\
\hline
\end{tabular}

beneficiosos controvertidos.

La hiperoxia producida por ECMO VA ocurre por la alta eficacia de los modernos oxigenadores y puede ser evitada reduciendo la $\mathrm{FiO}_{2}$ que pasa a través del filtro de oxigenación (gas de barrido) para mantener valores de $\mathrm{PaO}_{2}$ entre 60 y $100 \mathrm{mmHg}$. La eliminación de $\mathrm{CO}_{2}$ puede ser controlada incrementando el flujo total del gas de barrido a través del filtro de membrana en casos de acidosis respiratoria; disminuyendo el flujo si se presenta alcalosis[62]. Si el paciente se encuentra bajo ventilación mecánica se recomienda no usar Volumen Tidal alto o presión plateau $>25 \mathrm{~cm}$ $\mathrm{H}_{2} \mathrm{O}$ para disminuir el riesgo de barotrauma.

La descompresión del ventrículo izquierdo (VI) es un pilar fundamental en esta modalidad de ECMO para prevenir la lesión pulmonar secundaria a elevación de presiones venosas pulmonares, disminuyendo la estasis sanguínea en el VI y promueve la recuperación miocárdica[63]. En aquellos pacientes en los que a pesar de las medidas previamente mencionadas no se logra una adecuada descompresión ventricular, se deben emplear otras técnicas que aseguran descompresión ventricular, también llamadas técnicas de venteo o "venting".

La optimización del volumen durante la descompresión del VI con soporte es crucial, la optimización del estatus hídrico puede ser alcanzado mediante control de diuresis o terapias de reemplazo renal mediante un filtro de diálisis adaptado al circuito ECMO VA así evadiendo accesos vasculares adicionales que conllevarían a mayor riesgo de infecciones, trombosis o eventos hemorrágicos[64].

El ajuste de la terapéutica farmacológica en el paciente con soporte ECMO se debe realizar de manera dinámica ya que como describió Dzierbaa[65] se presentan grados variables de absorción por el tubo del circuito y el oxigenador modificando el volumen de distribución alterando los modelos farmacocinéticos y dinámicos especialmente para fármacos lipofilicos, sedantes, analgésicos y antimicrobianos.

El Síndrome de Arlequín (Norte-Sur) es una entidad característica que se puede presentar con soporte de esta modalidad del ECMO; en un contexto de intercambio pulmonar anormal de gases incluso cuando se combina con sangre completamente oxigenada de la cánula en la arteria femoral, la sangre que perfunde el cerebro, el corazón y las extremidades superiores pueden tener una saturación por debajo del $90 \%$ causando cianosis de la parte superior del cuerpo. Sorokin[66] y cols., recomiendan ajustar los parámetros ventilatorios, una canulación central, disminuir la eyección del VI o considerar ECMO V-AV (Figura 3) como estrategias que se pueden tomar para disminuir la incidencia de este fenómeno resaltando la importancia de los modelos híbridos.

Schmidt[67] y cols., estudiaron 3.846 pacientes con choque cardiogénico refractario manejados con ECMO VA identificando los principales factores que modifican la supervivencia; la insuficiencia renal crónica, una mayor duración de la ventilación mecánica antes del inicio de ECMO, las fallas orgánicas previas a ECMO, el paro cardíaco previo a ECMO, cardiopatías congénitas, presión de pulso y el bicarbonato sérico bajo fueron factores de riesgo asociados con la mortalidad. La edad más joven, menor peso, miocarditis aguda, trasplante de corazón, taquicardia o fibrilación ventricular refractaria, presión arterial diastólica elevada y presión inspiratoria pico más baja fueron protectores, creando una herramienta que predice la mortalidad de pacientes con choque cardiogénico refractario a tratamiento en 5 categorías correlacionado con la supervivencia post ECMO (ECMO SAVE Score) (Tabla 5).

\section{Indicaciones ECMO VA}

- Paro cardiaco (RCP Extracorpóreo o ECPR).

- Choque cardiogénico en el contexto de: 
- Síndrome coronario agudo.

- Insuficiencia cardiaca aguda.

- Falla ventricular derecha aguda debido a embolismo pulmonar.

- Falla ventricular derecha durante el uso de dispositivos de asistencia ventricular izquierda.

- Postcardiotomía incapaz de la salida del bypass cardiopulmonar.

- Falla primaria de injerto tras trasplante cardiaco.

- Miocarditis.

- Arritmias ventriculares refractarias.

- Hipotermia severa $\left(<28{ }^{\circ} \mathrm{C}\right)$ con inestabilidad hemodinámica.

- Miocardiopatía séptica.

Tabla 5. SAVE Score

\section{Parámetros}

Diagnóstico

- Miocarditis

- FV o TV refractaria

- Falla de injerto post trasplante cardiaco o pulmonar

- Cardiopatías congénitas

- Otros diagnósticos

Edad (años)

$-18-38$

$-39-52$

$-53-62$

$->63$

Peso $(\mathrm{Kg})$

$-<65$

$-65-89$

- >90

Falla orgánica aguda preECMO

- Falla hepática

- Disfunción del SNC

- Falla renal

- Enfermedad renal crónica (TFG $<60 \mathrm{ml}$.min.1,73 $\mathrm{m}^{2}$ por $>3$

meses)

Duración de intubado preECMO (horas)

$-<10$

$-11-29$

- > 30

Presión inspiratoria pico $<20 \mathrm{cmH}_{2} \mathrm{O}$

Paro cardiaco preECMO

Presión sanguínea diastólica preECMO $>40 \mathrm{mmHg}$

$\mathrm{HCO} 3$ preECMO $<15 \mathrm{mmol} / \mathrm{L}$

Valor constante para agregar a todos los cálculos

Total

Clasificación hospitalaria de supervivencia (SAVE Score)

$>5$

1 a 5

-4 a 0

-9 a -5

$<-10$

\section{Score}

3

$-3$

0

7

4

3

0

1

2

0

$-3$

$-3$

$-3$

$-6$

0

$-2$

$-4$

3

$-2$

3

$-3$

$-6$

-35 a 17

Clase

Supervivencia

75

58

II

42

III

30

IV

18

Schmidt y cols[54] 


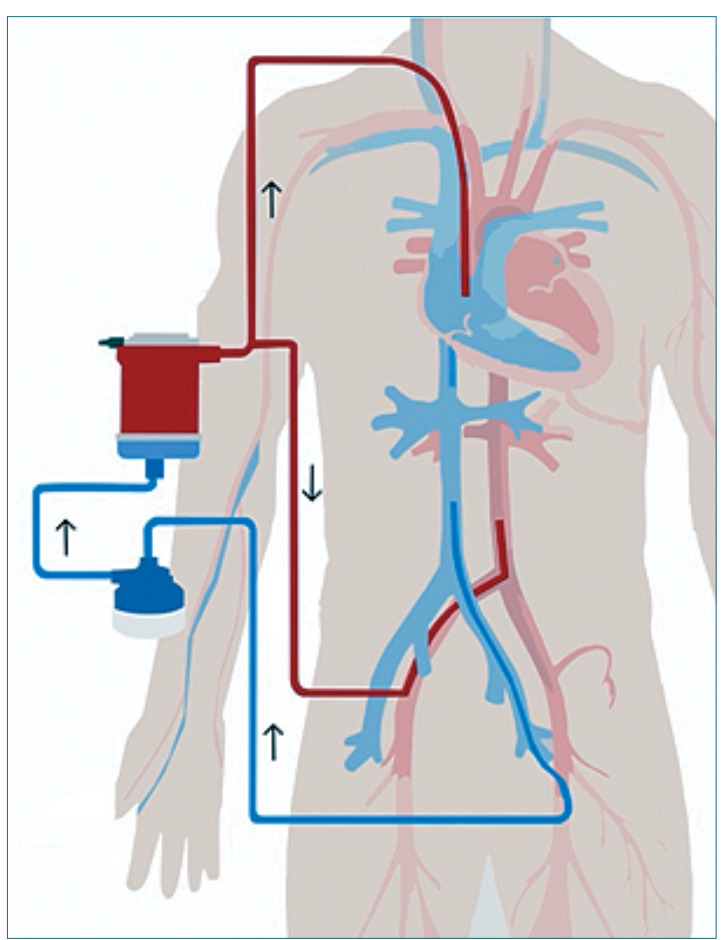

Figura 3. ECMO veno-arterio-venoso (hibrido).

- Intoxicación por drogas cardio toxicas.

Contraindicaciones ECMO VA
- Relativas

- Edad avanzada

- Coagulopatía severa o contraindicación a la anticoagulación (incluyendo enfermedad hepática avanzada).

- Acceso vascular limitado (obesidad extrema, extremidades amputadas).

- Absolutas

- Paro cardiaco no presenciado.

- Insuficiencia aortica severa.

- Falla multiorgánica severa e irreversible.

- Lesión cerebral severa irreversible.

- Enfermedad maligna diseminada.

- Disección aortica.

- Enfermedad arterial periférica (para canulación periférica).

- Baja probabilidad de recuperación miocárdica (excepto pacientes candidatos a trasplante cardiaco o soporte mecánico circulatorio duradero).

\section{Complicaciones}

La ausencia de ensayos controlados aleatorizados en México hace difícil determinar la prevalencia e incidencia de complicaciones de ECMO VA, a nivel internacional se han reportado una amplia gama de complicaciones; Keebler y cols., reportan hasta 20\%$30 \%$ de complicaciones vasculares, prevaleciendo la isquemia de extremidades en $40 \%$ seguido de hiperemia en 20\%. Masuzawa[73] describió una incidencia de 5 a $18 \%$ de hemolisis en pacientes con disposi-

\begin{tabular}{|c|c|c|c|}
\hline Referencia & Tipo de estudio & Población & Resultados \\
\hline Xie[68] y cols 2015 (CC) & Metaanálisis & $\begin{array}{l}\mathrm{n}=1.199 \text { (22 estudios), CC } \\
\mathrm{O} \text { PC }\end{array}$ & $\begin{array}{l}\text { Mortalidad Hospitalaria: } 60 \% \\
\text { (95\% IC: } 53 \%-66 \% \text { ) } \\
\text { Supervivencia: } 3 \text { meses 56\%, } 1 \\
\text { año } 54 \% \\
\text { Supervivencia a } 1 \text { año CC } 53 \% \\
\text { vs PR } 36 \%\end{array}$ \\
\hline $\begin{array}{l}\text { Cheng[69] y cols } 2014 \\
\text { (Miocarditis) }\end{array}$ & Metaanálisis & $\begin{array}{l}\mathrm{n}=170 \text { pacientes con mio- } \\
\text { carditis }\end{array}$ & $\begin{array}{l}\text { Mortalidad hospitalaria: 33\% } \\
\text { (95\% IC: } 26 \%-41 \%)\end{array}$ \\
\hline Batra[70] y cols 2016 & Cohorte retrospectiva & $\begin{array}{l}n=1.286 \text { con soporte ECMO } \\
\text { VA en Nueva York }\end{array}$ & $\begin{array}{l}\text { Mortalidad Hospitalaria: } 54 \% \\
\text { Supervivencia: } 1 \text { mes } 48 \%, 1 \\
\text { año } 38 \%\end{array}$ \\
\hline Aso[71] y cols 2016 & Cohorte retrospectiva & $\begin{array}{l}n=5.263 \text { con soporte } \\
\text { ECMO VA en Japón }\end{array}$ & $\begin{array}{l}\text { Mortalidad: } 73 \% \text { (choque } 74 \% \text {, } \\
\text { embolismo pulmonar } 64 \% \text { ) }\end{array}$ \\
\hline $\begin{array}{l}\text { D'Alessandro[72] y cols. } 2010 \\
\text { (Trasplante cardiaco) }\end{array}$ & Cohorte retrospectiva & $\begin{array}{l}\mathrm{n}=54 \text { pacientes con falla } \\
\text { aguda del injerto por cual- } \\
\text { quier causa }\end{array}$ & $\begin{array}{l}\text { Mortalidad: } 50 \% \\
\text { Supervivencia: } 1 \text { año } 73 \%\end{array}$ \\
\hline
\end{tabular}

CC: Choque cardiogénico; PR: Paro cardiaco; IC: Intervalo de confianza. 
tivo de asistencia ventricular adaptando una bomba de sangre centrífuga suspendida magnéticamente con un motor auto portable; la hemoglobina libre de plasma > $100 \mathrm{mg} / \mathrm{L}$ (indicador de hemolisis) se elevó hasta en $67 \%$ de los casos asociándose a un mayor riesgo de mortalidad (32\%). Respecto a complicaciones renales; la insuficiencia renal aguda es reportada con una incidencia del 33\% al 55,6\%, sin diferencia significativa respecto al tipo de canulación, la prevalencia de hemodiálisis post ECMO VA es reportada entre $28 \%$ y $52 \%[74],[75]$. Los resultados en la prevalencia de sangrado y hemorragia son muy variables en la literatura internacional por no haber una estandarización de definiciones, 10 eventos por cada 100 días de ECMO, los sitios más comunes son tórax, sitio de cánulas y tracto gastrointestinal[76]. Una manifestación interesante observada en el estudio The ENCOURAGE fue la presencia síndrome de respuesta inflamatoria sistémica hasta en $30 \%$ de los casos después de la decanulación asociado a factores de riesgo como edad > 60 años, mayor duración de ECMO e infección[77],[78]. La prevalencia de infección sanguínea va del $3 \%$ al $18 \%$ e incidencia de 2,98 a 20,55 episodios por 1.000 días de ECMO en adultos; la incidencia de infecciones del tracto respiratorio inferior se informa en 24,4 episodios por 1.000 días de ECMO y la prevalencia de infecciones del tracto urinario se reporta entre $1 \%$ y $2 \%$, con una incidencia de 1 a 13,8 casos por 1.000 días de ECMO.

\section{ECMO en México}

En México, el uso y desarrollo de ECMO ha sido mermado, debido a la escasez de recursos y falta organización estructural en el Sector Salud. Diferentes autores mexicanos como Careaga[54] y cols., han desarrollado reporte de casos con resultados satisfactorios. León Ramírez[55] empleo ECMO VA como terapia puente para trasplante cardiaco en un masculino de 62 años de edad, con antecedente de enfermedad de Chagas con fallo ventricular severo y con evolución satisfactoria postquirúrgico. Garza[56] y cols., reportaron el primer manejo exitoso de hernia diafragmática congénita con ECMO VV de un paciente masculino de 37,5 semanas de gestación. No existen por el momento en nuestro país grandes estudios controlados y aleatorizados que reflejen de manera verídica los números de ECMO en función a sus resultados, indicaciones, complicaciones y mortalidad. ECMO VV y VA son usados para tratamiento de falla respiratoria aguda con la diferencia de que el segundo puede proveer soporte circulatorio completo.
La Utilidad del ECMO veno-arterial en el manejo posquirúrgico del choque cardiogénico refractario en enfermedad aórtica compleja de una paciente femenina de 38 años con Síndrome de Marfan fue descrito por Rodríguez-Hernández y cols., en 2018, dos años después de su egreso la paciente se reportó en fase III de rehabilitación cardiaca y clase funcional I, FEVI $56 \%$, cavidades cardiacas sin dilatación, movilidad global y segmentaria en reposo normal y prótesis aortica funcional.

ECMO es una terapia que se ha usado con mayor o menor éxito, desde hace varios años por diferentes grupos siendo pionero en esta, el Dr. Rene Gómez del grupo de Monterrey (Centro ELSO 321), actualmente es el vicepresidente de ELSO LATAM y que se ha encargado de impulsar el uso correcto de esta tecnología por medio de cursos que actualmente son de gran relevancia en el país. La incorporación de nuevas maquinas, cánulas, técnicas de canulación y la organización de grupos multidisciplinarios que usan este tipo de dispositivos de forma mas o menos rutinaria es relativamente reciente.

La utilización de este tipo de equipo, demanda una preparación sumamente puntual y compleja, muchos de los grupos que en México están desarrollando este tipo de terapia han asistido a cursos presenciales en otros países en Europa, Estados Unidos, Canadá, Latinoamérica, etc., quienes, por el tipo de sistema de salud, han logrado desarrollar un nivel de experiencia mucho mayor al de nuestro país.

Una vez que un grupo de médicos, habitualmente cirujanos vasculares o cardiovasculares, anestesiólogos cardiovasculares, intensivistas, cardiólogos, neumólogos, perfusionistas, enfermeras, paramédicos, etc., se han capacitado en algún centro con experiencia, viene la parte de desarrollar la experiencia propia, tratar de cometer la menor cantidad de errores durante el proceso y poder ofrecer la mejor calidad de atención al paciente mas críticamente enfermo que existe en el hospital. Hay muchas guías y sugerencias para desarrollar un "ECMO team" y un programa de atención de pacientes críticos con ECMO, sin embargo, todas ellas deben adaptarse a la realidad y a los recursos con que se cuenta en cada hospital, tanto recursos materiales como humanos, solo en ese momento va a poder iniciarse la canulación y entrada en ECMO del primer paciente de cada hospital. Sin duda es una de las experiencias mas increíbles el poder tener a un paciente sin función respiratoria y/o cardiaca y poder mantenerlo vivo mientras esperamos que el problema que lo llevo a dicha disfunción mejore y logremos retirar este dispositivo lo mas rápido posible o bien se pueda llevar con éxito a un trasplante 
cardiaco o pulmonar. Conforme la cantidad de pacientes atendidos se incrementa, la experiencia y la sistematización de muchos procesos va mejorando y se obtienen mejores resultados, a continuación, sigue la preparación de mas personal intrahospitalario que esta en contacto con este tipo de pacientes, transmitir la experiencia propia y tratar de involucrar a mas personas de diferentes áreas sin duda es una tarea muy difícil pero que después de un tiempo es imprescindible y da resultados fenomenales. Nadie que este en contacto con un paciente en ECMO puede estar sin haberse preparado ya sea con el grupo institucional o en cursos ajenos al hospital.

Después del ensayo CESAR (soporte ventilatorio convencional versus oxigenación por membrana extracorpórea para pacientes con SIRA severo), en el que ECMO parecía ser superior al ventilador en la población adulta, generó que aumentara su uso en el mundo y en México. En el hospital de Cardiología del Centro Medico Nacional siglo XXI (Centro ELSO 673), se inició esta terapia en el año 2012, bajo la dirección del Dr. Moisés Calderón, durante cerca de 5 años se colocaron alrededor de 43 casos, la mayoría de tipo veno - arterial en choque postcardiotomía con resultados negativos al inicio. En 2015, Meza y cols., reseñaron en una carta científica el uso de ECMO VA en un paciente masculino de 33 años con diagnóstico de hipertensión pulmonar tromboembolica crónica con fallo del ventrículo derecho operado de tromboendarterectomía pulmonar con efectos benéficos, fue hasta el año 2017 cuando el programa a cargo del Dr. Carlos Riera Kinkel toma un giro completo, se establecen protocolos, se entrena a todo el equipo medico y paramédico, se establece un programa de educación continua para médicos y enfermeras, se inicia la formación de un ECMO team, con ello los resultados mejoraron considerablemente, se colocaron cerca de 40 casos en ECMO con una sobrevida de $52,7 \%$, se comenzó a variar el diagnostico de utilización en la cual se utilizaba ecmo en falla respiratoria, falla cardiaca por miocarditis y miocardiopatía dilatada, por isquemia y choque postcardiotomia, se realizaron también los primeros 3 casos de ECMO móviles registrados en una institución publica. Se colocaron estos dispositivos tanto en adultos como en pacientes pediátricos. Sin duda fue un parteaguas para el desarrollo de equipo multidisciplinario, se realizaron incluso soportes simultáneos con ECMO, hasta 4 en una ocasión, se adquirieron mas consolas de ECMO de diferentes marcas, lo cual permitió al grupo mantenerse a la vanguardia mundial.

A la par, parte del grupo del Hospital de Cardiología se unió a grupos en instituciones privadas que brindan terapia ECMO como en el Hospital Centro Medico ABC (Centro ELSO 778), donde gracias a esta experiencia desarrollada en el Hospital de Cardiología hoy el grupo privado $A B C$ ha tenido mejores resultados en la sobrevida de sus casos conectados a ECMO al trabajar de la mano con nuestro grupo.

Actualmente, en México existen diferentes grupos con mayor o menor experiencia en el uso de este tipo de tecnología y los resultados cada vez mas cercanos a los reportados internacionalmente, nuestro grupo se ha desarrollado en uno de los principales hospitales de atención de patologías cardiacas en México, la unidad medica de alta especialidad de cardiología, en Centro Médico Nacional siglo XXI, dado que es un hospital de concentración, la cantidad y complejidad de pacientes que se atienden suele ser muy alta, normalmente podemos tener incluso mas de 10 cirugías cardiacas al día en pacientes con diferentes comorbilidades mas o menos criticas y que dependiendo del tipo de cirugía al que se exponen pueden llegar a requerir algún tipo de soporte circulatorio.

En Guadalajara, México se formo a mediados de 2015 (Centro ELSO 742), un grupo multidisciplinario con la integración de líderes en el campo de los cuidados críticos cardiovasculares y de la terapia intensiva, entrenados mayormente en las escuelas europeas de más experiencia (Alemania, Francia, España e Italia) así como en importantes centros de Estados Unidos. Actualmente, han expandido su presencia a la ciudad de Chihuahua, con la adhesión de un equipo de intensivistas líderes, además de lograr alianzas estratégicas con grupos en las ciudades de Puebla, León, Tijuana, Ciudad Juárez, Tijuana entre otras. Al ser un grupo integrado por médicos que laboran en el medio privado cuentan con gran experiencia en la optimización de recursos en sus casos al realizar alianzas con las principales compañías de seguros nacionales e internacionales que den la cobertura suficiente durante la terapia de nuestros pacientes. Al igual que otros grupos en el país, como es mandatorio, el grupo de Guadalajara participa activamente en la capacitación continua del personal médico y paramédico de los centros ELSO a su cargo, así como en múltiples congresos nacionales e internacionales, compartiendo experiencias con otros líderes de distintas latitudes y enriqueciendo a la vez nuestro acervo clínico y académico. Así, el grupo de Guadalajara participa en el trabajo de la asistencia extracorpórea con altos estándares de calidad siempre dispuestos a colaborar con los diferentes grupos que formamos esta gran familia dedicada a la asistencia circulatoria y respiratoria extracorpórea.

El crecimiento de esta terapia en México cada vez 
es mayor, tenemos la fortuna de contar con grupos de médicos con mucho interés en incorporar esta terapia a su armamento para el tratamiento de pacientes críticos, la forma en que esto puede desarrollarse y que va a atenuar el riesgo de exponer al paciente a la curva de aprendizaje de cada grupo, es apoyarse en grupos que ya tienen cierto grado de experiencia con el uso de esta tecnología para ir afinando detalles y resolviendo las eventualidades inesperadas que vienen con su uso. De esta manera, los resultados van a ser cada vez mejores y se hará mas cotidiano el uso de este tipo de dispositivos en diferentes escenarios en el país, esta es una metodología comprobada por nuestro grupo que tuvo la oportunidad de colaborar con un grupo nuevo en otro estado del país y se pudo sacar adelante a un paciente con hemorragia alveolar que requirió soporte con ECMO VV, ambos grupos trabajamos en estrecha comunicación y colaboración logrando colocar, mantener y destetar con éxito al paciente tras mas dos semanas de tratamiento.

La sistematización del uso de esta terapia en México aun es un tema pendiente, poder ajustar e incorporar las guías y recomendaciones internacionales a nuestra realidad y al sistema de salud tanto privado como publico sin duda es un gran reto pero con la colaboración de los diferentes grupos del país, se podrá ir delineando cada vez más, la sistematización de los procesos, generación de evidencia científica de nuestra población y guías propias que lleven a un uso mas eficiente de dispositivos en nuestro país, poder tener cada vez mejores resultados para el paciente mas critico que puede haber en un hospital.

\section{Conclusiones}

En la actualidad, el auge tecnológico de los dispositivos biomédicos en el campo de ECMO VA y VV, ha tenido un gran impacto en la sobrevida de los pacientes críticamente enfermos y su manejo. El progreso de esta terapéutica en nuestro país se ha visto preso por cuestiones económicas, dado que sigue siendo una terapia de alto costo y por pobres políticas de salud publica, que limitan la expansión de uso de este tipo de terapia, en el sistema de salud privado en México, aun los seguros de gastos médicos, suelen tener mucha resistencia a la cobertura de esta tecnología. Resulta vital la investigación, crecimiento y desarrollo de evidencia científica mexicana, para ofrecerle a nuestros pacientes una alternativa de tratamiento y esperanza de vida. El ECMO es una terapia de uso habitual para pacientes con falla cardiovascular o respiratoria grave, utilizada mayormente en Norteamérica y Europa, sin embargo, en México es relativamente nueva, con muy pocos centros especializados y muy poco personal de salud entrenado al respecto, por lo que la unificación de criterios y sistematización de procesos, comienza a ser parte de un futuro prometedor.

\section{Referencias}

1. MacLaren G, Combes A, Bartlett $\mathrm{RH}$. Contemporary extracorporeal membrane oxygenation for adult respiratory failure: life support in the new era. Intensive Care Med 2012; 38:210-20. https://doi.org/10.1007/s00134011-2439-2

2. Shekar K, Mullany DV, Thomson $B$, et al. Extracorporeal life support devices and strategies for management of acute cardiorespiratory failure in adult patients: a comprehensive review. Crit Care 2014; 18:219. https://doi. org/10.1186/cc13865

3. Peek GJ, Mugford M, Tiruvoipati $R$, et al. Efficacy and economic assessment of conventional ventilatory support versus extracorporeal membrane oxygenation forsevere adult respiratory failure (CESAR): a multicenter randomised controlled trial. Lancet 2009; 374:1351-63. https://doi.org/10.1016/S01406736(09)61069-2

4. Cooper DS, Jacobs JP, Moore L, et al. Cardiac extracorporeal life support: state of the art in 2007. Cardiol Young 2007;17 Suppl 2:104-15. https://doi.org/10.1017/ S1047951107001217

5. Marasco SF, Esmore DS, Negri $J$, et al. Early institution of mechanical support improves outcomes in primary cardiac allograft failure. J Heart Lung Transplant 2005; 24:2037-42. https://doi.org/10.1016/j.healun.2005.06.007

6. Acker MA. Mechanical circulatory support for patients with acute-fulminant myocarditis. Ann Thorac Surg 2001;71: S73-6; discussion S82-5. https://doi.org/10.1016/S00034975(00)02628-X

7. Clark JB, Pauliks LB, Myers JL, et al. Mechanical circulatory support for end-stage heart failure in repaired and palliated congenital heart disease. Curr Cardiol Rev 2011; 7:102-9. https://doi./1 $0.2174 / 157340311797484222$

8. Rinieri P, Peillon C, Bessou JP, et al. National review of use of 
extracorporeal membrane oxygenation as respiratory support in thoracic surgery excluding lung transplantation. Eur J Cardiothorac Surg 2015; 47:87-94. https:// doi.org/10.1093/ejcts/ezu127

9. McFadden PM, Greene CL. The evolution of intraoperative support in lung transplantation: Cardiopulmonary bypass to extracorporeal membrane oxygenation. J Thorac Cardiovasc Surg 2015; 149:1158-60. https://doi. org/10.1016/j.jtcvs.2014.12.020

10. Gulack BC, Hirji SA, Hartwig MG. Bridge to lung transplantation and rescue post-transplant: the expanding role of extracorporeal membrane oxygenation. J Thorac Dis 2014; 6:1070-9.

11. Bermudez CA, Shiose A, Esper SA, et al. Outcomes of intraoperative venoarterial extracorporeal membrane oxygenation versus cardiopulmonary bypass during lung transplantation. Ann Thorac Surg 2014; 98:193642; discussion 1942-3. https:// doi.org/10.1016/j.athoracsur.2014.06.072

12. Pham $T$, Combes $A$, Rozé $H$, et al. Extracorporeal membrane oxygenation for pandemic influenza $A(\mathrm{H} 1 \mathrm{~N} 1)$-induced acute respiratory distress syndrome: a cohort study and propensitymatched analysis. Am J Respir Crit Care Med 2013; 187:27685. https://doi.org/10.1164/ rccm.201205-08150C

13. Combes $A$, Leprince $P$, Luyt $C E$, et al. Outcomes and longterm quality-of-life of patients supported by extracorporeal membrane oxygenation for refractory cardiogenic shock. Crit Care Med 2008; 36:140411. https://doi.org/10.1097/ CCM.0b013e31816f7cf7

14. Bréchot $N$, Luyt $C E$, Schmidt $M$, et al. Venoarterial extracorporeal membrane oxygenation support for refractory cardio- vascular dysfunction during severe bacterial septic shock. Crit Care Med 2013; 41:161626. https://doi.org/10.1097/ CCM.0b013e31828a2370

15. Fraser JF, Shekar K, Diab S, et al. ECMO - the clinician's view. ISBT Sci Ser 2012; 7:82-8. https://doi.org/10.1111/j.17512824.2012.01560.x

16. Kolff WJ, Berk HT, ter Welle $M$, et al. The artificial kidney: a dialyser with a great area. 1944. J Am Soc Nephrol 1997; 8:195965.

17. Gibbon JH Jr. Application of a mechanical heart and lungmapparatus to cardiac surgery. Minn Med 1954; 37:171-85; passim.

18. Rashkind WJ, Freeman A, Klein $D$, et al. Evaluation of a disposable plastic, low volume, pumpless oxygenator as a lung substitute. J Pediatr 1965; 66:94102. https://doi.org/10.1016/ S0022-3476(65)80342-0

19. Dorson W Jr, Baker E, Cohen $\mathrm{ML}$, et al. A perfusion system for infants. Trans Am Soc Artif Intern Organs 1969; 15:155-60.

20. Baffes TG, Fridman JL, Bicoff JP, et al. Extracorporeal circulation for support of palliative cardiac surgery in infants. Ann Thorac Surg 1970; 10:354-63. https://doi.org/10.1016/S00034975(10)65613-5

21. Hill JD, O'Brien TG, Murray JJ, et al. Prolonged extracorporeal oxygenation for acute posttraumatic respiratory failure (shock-lung syndrome). Use of the Bramson membrane lung. N Engl J Med 1972; 286:62934. https://doi.org/10.1056/ NEJM197203232861204

22. Zapol WM, Kitz RJ (1972) Buying time with artifi cial lungs. N Engl J Med 286:657658. https://doi.org/10.1056/ NEJM197203232861210

23. Kolobow T, Gattinoni L,
Tomlinson T, et al. The carbon dioxide membrane lung (CDML): a new concept. Trans Am Soc Artif Intern Organs 1977; 23:17-21. https://doi. org/10.1097/00002480197700230-00005

24. Bartlett RH, Gazzaniga $A B$, Jefferies MR, et al. Extracorporeal membrane oxygenation (ECMO) cardiopulmonary support in infancy. Trans Am Soc Artif Intern Organs 1976; 22:80-93

25. Morris AH, Wallace CJ, Menlove $\mathrm{RL}$, et al. Randomized clinical trial of pressure-controlled inverse ratio ventilation and extracorporeal $\mathrm{CO} 2$ removal for adult respiratory distress syndrome. Am J Respir Crit Care Med 1994; 149:295305. https://doi.org/10.1164/ ajrccm.149.2.8306022

26. Lewandowski K, Rossaint R, Pappert $D$, et al. High survival rate in 122 ARDS patients managed according to a clinical algorithm including extracorporeal membrane oxygenation. Intensive Care Med 1997; 23:819-35. https://doi.org/10.1007/ s001340050418

27. Kolla S, Awad SS, Rich PB, et al. Extracorporeal life support for 100 adult patients with severe respiratory failure. Ann Surg 1997; 226:54464; discussion 565-6. https:// doi.org/10.1097/00000658199710000-00015

28. Bartlett RH, Roloff DW, Custer $J R$, et al. Extracorporeal life support: The University of Michigan experience. JAMA 2000; 283:904-8. https://doi. org/10.1001/jama.283.7.904

29. Combes A, Hajage D, CapeIlier G, Demoule A, Lavoue S, Guervilly $C$, et al. Extracorporeal Membrane Oxygenation for Severe Acute Respiratory Distress Syndrome. NEngl J Med 2018; 378:1965-75. https://doi. 
org/10.1056/NEJMoa1800385

30. Ashbaugh DG, Bigelow DB, Petty TL, Levine BE. Acute respiratory distress in adults. Lancet 1967; 2:319-23. https:// doi.org/10.1016/S01406736(67)90168-7

31. Ware LB, Matthay MA. The acute respiratory distress syndrome. N Engl J Med 2000; 342:133449. https://doi.org/10.1056/ NEJM200005043421806

32. Bernard, G.R.; Artigas, A.; Brigham, K.L.; Carlet, J.; Falke, K.; Hudson, L.; Lamy, M.; Legall, J.R.; Morris, A.; Spragg, R. The american-european consensus conference on ards.

33. Munshi L, Telesnicki T, Walkey A, et al. Extracorporeal life support for acute respiratory failure. A systematic review and metaanalysis. Annals ATS 2014; 11:802-10. https://doi. org/10.1513/AnnalsATS.201401$0120 \mathrm{C}$

34. Zampieri FG, Mendes PV, Ranzani OT, et al. Extracorporeal membrane oxygenation for severe respiratory failure in adult patients: a systematic review and metaanalysis of current evidence. J Crit Care 2013; 28: 9981005. https://doi.org/10.1016/j. jcrc.2013.07.047

35. Davies A, Jones D, Bailey M, et al. Extracorporeal membrane oxygenation for 2009 influenza $\mathrm{A}(\mathrm{H} 1 \mathrm{~N} 1)$ acute respiratoy distress syndrome. JAMA 2009; 302:1888-95. https://doi. org/10.1001/jama.2009.1535

36. Noah MA, Peek GJ, Finney SJ, et al. Referral to an extracorporeal membrane oxygenation center and mortality among patients with severe 2009 influenza A (H1N1). JAMA 2011; 306:165968. https://doi.org/10.1001/ jama.2011.1471

37. Pham $\mathrm{T}$, Combes A, Roze $\mathrm{H}$, et al. Extracorporeal membrane oxygenation for pandemic in- fluenza A (H1N1)-induced acute respiratory: a cohort study and propensity-matched analysis. Am J Resp Crit Care Med 2013; 187: 276-85. https://doi.org/10.1164/ rccm.201205-08150C

38. Sameed M, Meng Z, Marciniak ET. EOLIA trial: the future of extracorporeal membrane oxygenation in acute respiratory distress syndrome therapy? Breathe 2019; 15: 244-246. https://doi. org/10.1183/20734735.03632018

39. Griffiths MJD, McAuley DF, Perkins GD, et al. Guidelines on the management of acute respiratory distress syndrome. BMJ Open Resp Res 2019;6: e000420. https://doi.org/10.1136/ bmiresp-2019-000420

40. Fan E, Gattinoni L, Combes A, Schmidt M, Peek G, Brodie D, et al. Venovenous extracorporeal membrane oxygenation for acute respiratory failure. Intensive Care Med (2016) 42:712-724. https://doi.org/10.1007/s00134016-4314-7

41. Barbaro RP, Odetola FO, Kidwell KM et al (2015) Association of hospital-level volume of extracorporeal membrane oxygenation cases and mortality. Analysis of the Extracorporeal Life Support Organization registry. Am J Respir Crit Care Med 191:894901. https://doi.org/10.1164/ rccm.201409-16340C

42. Combes A, Brodie D, Bartlett R et al (2014) Position paper for the organization of extracorporeal membrane oxygenation programs for acute respiratory failure in adult patients. Am J Respir Crit Care Med 190:488496. https://doi.org/10.1164/ rccm.201404-0630CP

43. Rastan AJ, Lachmann N, Walther T et al (2006) Autopsy findings in patients on postcardiotomy extracorporeal membrane oxygenation (ECMO). Int J Artif Organs
29:1121-1131. https://doi./10.11 77/039139880602901205

44. Combes A, Leprince $P$, Luyt C-E et al (2008) Outcomes and long-term quality-of-life of patients supported by extracorporeal membrane oxygenation for refractory cardiogenic shock. Crit Care Med 36:14041411. https://doi.org/10.1097/ CCM.0b013e31816f7cf7

45. Cooper E, Burns J, Retter A et al (2015) Prevalence of venous thrombosis following venovenous extracorporeal membrane oxygenation in patients with severe respiratory failure. Crit Care Med 43: e581-e584. https://doi.org/10.1097/ CCM.0000000000001277

46. Lubnow M, Philipp A, Foltan M et al (2014) Technical complications during veno-venous extracorporeal membrane oxygenation and their relevance predicting a system-exchangeretrospective analysis of 265 cases. PLoS One 9: e112316. https://doi.org/10.1371/journal. pone. 0112316

47. Müller T, Lubnow M, Philipp A et al (2011) Risk of circuit infection in septic patients on extracorporeal membrane oxygenation: a preliminary study. Artif Organs 35: E84-E90. https://doi.org/10.1111/j.15251594.2010.01185.x

48. Glick D, Dzierba AL, Abrams D et al (2015) Clinically suspected heparin induced thrombocytopenia during extracorporeal membrane oxygenation. J Crit Care 30:1190-1194. https://doi. org/10.1016/j.jcrc.2015.07.030

49. Pan KC, McKenzie DP, Pellegrino $\checkmark$ et al (2015) The meaning of a high plasma free haemoglobin: retrospective review of the prevalence of haemolysis and circuit thrombosis in an adult ECMO centre over 5 years. Perfusion. https://doi. 
rg/10.1177/0267659115595282

50. Weingart C, Lubnow M, Philipp A et al (2015) Comparison of coagulation parameters, anticoagulation, and need for transfusion in patients on interventional lung assist or veno-venous extracorporeal membrane oxygenation. Artif Organs 39:765773. https://doi.org/10.1111/ aor. 12464

51. Mary E. Keebler, Elias V. Haddad, Chun W. Choi, Stuart McGrane. Venoarterial Extracorporeal Membrane Oxygenation in Cardiogenic Shock. JACC: Heart failure. American College of cardiology foundation. 2018; 6: 503-516. https://doi. org/10.1016/j.jchf.2017.11.017

52. Batra J, Toyoda N, Goldstone AB, Itagaki S, Egorova NN, Chikwe J. Extracorporeal membrane oxygenation in New York State: trends, outcomes, and implications for patient selection. Circ Heart Fail 2016;9: e003179. https://doi.org/10.1161/CIRCHEARTFAILURE. 116.003179

53. Gerke AK, Tang F, Cavanaugh $J E$, Doerschug KC, Polgreen PM. Increased trend in extracorporeal membrane oxygenation use by adults in the United States since 2007. BMC Res Notes 2015;8: 686. https://doi.org/10.1186/ s13104-015-1678-7

54. Careaga Reyna Guillermo, Carlos Alberto Lezama Urtecho, Violeta Denice Valencia Santoyo, Jaime Zaldívar Cervera. Oxigenación extracorpórea con membrana (ECMO), en una paciente con insuficiencia respiratoria aguda por influenza A. Neumologia y cirugia de Torax. 2009: 68;3.

55. León-Ramírez, Santiago López J. Oxigenación por membrana extracorpórea como puente para trasplante cardiaco por miocardiopatía chagásica. Revista Médica del Instituto Mexicano del Seguro Social. 2018:6; 429-
433.

56. Garza-Serna U, Gómez-Gutiérrez R, Quezada-Valenzuela G, Cárdenas-Del Castillo B, Contreras-Cepeda V, Chávez-García E, Nieto-Sanjuanero A. Manejo exitoso de hernia diafragmática congénita con ECMO. Primer reporte de caso en México. Acta Pediatr Mex. 2017;6: 378-385. https://doi.org/10.18233/APM38No6pp378-3851505

57. Luis Raúl Meza-López, Luis Efren Santos-Martínez, Víctor Manuel Lozano-Torres, Rutilio Daniel Jiménez-Espinosa, Jesus Zaín Campos-Larios. Uso de oxigenador de membrana extracorpórea venoarterial en un paciente con fallo del ventrículo derecho operado de tromboendarterectomía pulmonar. Carta científica (2015). https://doi.org/10.1016/j. acmx.2014.11.001

58. Alejandra Rodríguez-Hernández, Carlos Adán-Brito, Gustavo Rojas-Velasco, Francisco Martín Baranda-Tovar. Utilidad del ECMO veno-arterial en el manejo posquirúrgico del choque cardiogénico refractario en enfermedad aórtica compleja. Archivo Cardiol Mex. 2018; 88: 321-325. https://doi.org/10.1016/j. acmx.2017.07.001

59. Peek GJ, Mugford M, Tiruvoipati $\mathrm{R}$, et al., CESA trial collaboration. Efficacy and economic assessment of conventional ventilatory support versus extracorporeal membrane oxygenation for severe adult respiratory failure (CESAR): a multicentre randomised controlled trial. Lancet 2009;374: 1351-63. https://doi.org/10.1016/S01406736(09)61069-2

60. Maya Guglin, Mark J. Zucker, Vanessa M. Bazan. Venoarterial ECMO for Adults. JAAC: Scientific expert panel. 2019: 6.

61. Silvetti S, Koster A, Pappalardo
F. Do we need heparin coating for extracorporeal membrane oxygenation? New concepts and controversial positions about coating surfaces of extracorporeal circuits. Artif Organs 2015; 39:176-9. https://doi. org/10.1111/aor.12335

62. Jaski BE, Ortiz B, Alla KR, et al. A 20-year experience with urgent percutaneous cardiopulmonary bypass for salvage of potential survivors of refractory cardiovascular collapse.

63. Meani P, Gelsomino S, Natour $E$, et al. Modalities and effects of left ventricle unloading on extracorporeal life support: a review of the current literature. Eur J Heart Fail 2017;19 Suppl 2:84-91. https://doi.org/10.1002/ ejhf.850

64. Chen H, Yu RG, Yin NN, Zhou JX. Combination of extracorporeal membrane oxygenation and continuous renal replacement therapy in critically ill patients: a systematic review. Crit Care 2014;18: 675. https://doi. org/10.1186/s13054-014-0675-x

65. Dzierba AL, Abrams D, Brodie D. Medicating patients during extracorporeal membrane oxygenation: the evidence is building. Crit Care 2017; 21:66. https:// doi.org/10.1186/s13054-0171644-y

66. Sorokin V, MacLaren G, Vidanapathirana PC, Delnoij T, Lorusso R. Choosing the appropriate configuration and cannulation strategies for extracorporeal membrane oxygenation: the potential dynamic process of organ support and importance of hybrid modes. Eur J Heart Fail 2017; 19 Suppl 2:75-83. https:// doi.org/10.1002/ejhf.849

67. Schmidt M, Burrell A, Roberts $L$, et al. Predicting survival after ECMO for refractory cardiogenic shock: the survival after venoarterial-ECMO (SAVE)-score. 
Eur Heart J 2015; 36:2246-56. https://doi.org/10.1093/eurheartj/ehv194

68. Xie A, Phan K, Tsai YC, Yan TD, Forrest $P$. Venoarterial extracorporeal membrane oxygenation for cardiogenic shock and cardiac arrest: a meta-analysis. J Cardiothorac Vasc Anesth. 2015; 29:637-45. https://doi. org/10.1053/j.jvca.2014.09.005

69. Cheng R, Hachamovitch R, Kittleson M, Patel J. Clinical outcomes in fulminant myocarditis requiring extracorporeal membrane oxygenation: a weighted meta-analysis of 170 patients. J Card Fail. 2014;20(6):4006. https://doi.org/10.1016/j. cardfail.2014.03.005

70. Batra J, Toyoda N, Goldstone AB, Itagaki S. Extracorporeal Membrane Oxygenation in New York State: Trends, Outcomes, and Implications for Patient Selection. Circ Heart Fail. 2016;9(12). https://doi.org/10.1161/CIRCHEARTFAILURE.116.003179

71. Shotaro Aso. Hiroki M. Kiyohide F. Hideo Y. In-hospital mortality and successful weaning from venoarterial extracorporeal membrane oxygenation: analysis of 5,263 patients using a national inpatient database in Japan. Critical Care volume 2016; 20: 80. https://doi.org/10.1186/s13054016-1261-1

72. D'Alessandro C, Aubert S, Golmard JL, Praschker BL, Luyt CE, Pavie A, Gandjbakhch I, Leprince P. Extra-corporeal membrane oxygenation temporary support for early graft failure after cardiac transplantation. Eur J Cardiothorac Surg. 2010;37(2):3439. https://doi.org/10.1016/j. ejcts.2009.05.034

73. Masuzawa T, Onuma H, Kim SJ, Okada Y. Magnetically suspended centrifugal blood pump with a self-bearing motor. ASAIO J 2002;48: 437-42. https:// doi.org/10.1097/00002480200207000-00019

74. Sanfilippo F, Asmussen S, Maybauer DM, et al. Bivalirudin for alternative anticoagulation in extracorporeal membrane oxygenation: a systematic review. J Intens Care Med 2017; 32:312-9. https://doi. rg/10.1177/0885066616656333

75. Thiagarajan RR, Barbaro RP, Rycus PT, et al. Extracorporeal Life Support Organization Registry International Report 2016. ASAIO J 2017; 63:60-

7. https://doi.org/10.1097/ MAT.0000000000000475

76. Johnson NJ, Acker M, Hsu CH, et al. Extracorporeal life support as rescue strategy for outof- hospital and emergency department cardiac arrest. Resuscitation 2014; 85:1527-32. https:// doi.org/10.1016/j.resuscitation.2014.08.028

77. Muller $G$, Flecher E, Lebreton $G$, et al. The ENCOURAGE mortality risk score and analysis of longterm outcomes after VA-ECMO for acute myocardial infarction with cardiogenic shock. Intens Care Med 2016; 42:370-8. https://doi.org/10.1007/s00134016-4223-9

78. Bartlett RH. John H Gibbon Jr Lecture. Extracorporeal life support: Gibbon fulfilled. J Am Coll Surg 2014; 218:317-27. https://doi.org/10.1016/j.jamcollsurg.2013.12.002 